\title{
Experiencia piloto con esquema rifapentina-isoniazida semanal por 3 meses para tratamiento de la infección tuberculosa latente en el Programa Nacional de Tuberculosis de Chile
}

TANIA HERRERA M.*, NADIA ESCOBAR S.* y NATALIA RUIZ L.*

Pilot experience with weekly rifapentine-isoniazid treatment for 3 months for latent tuberculosis infection at the Tuberculosis National Program of Chile

Introduction: Prevention of active tuberculosis in risk groups is crucial in tuberculosis control and elimination. Treatment of latent tuberculosis (TITL) with rifapentine and isoniazid in weekly doses for 12 weeks is shorter than other pharmacological treatments, with less liver toxicity, better patient compliance and it is cost-effective. The objective of this study is to evaluate the feasibility to implement this treatment at a programmatic level in Chile. Methods: A pilot intervention was conducted in selected territories between May 2018 and March 2019. Within these territories, the regulated treatment with isoniazid 6 months was replaced by the 12 weeks treatment with weekly rifapentine-isoniazide. Additionally, the target population was expanded to include contacts over 14 years old, currently not included in the national guidelines. Treatment consisted in oral administration of rifapentine and isoniazide together once a week for 12 weeks, under supervision of trained health workers. Results: From 238 patients entered to the protocol, 53\% of them were women and $54.2 \%$ were older than 14 years-old. Out of the total number of patients, 203 (85.3\%) completed treatment, 22 (9.2\%) abandoned, 8 (3.4\%) had adverse drug reactions, and 5 ended treatment for different causes. Conclusion: Both TITL with rifapentine-isoniazide in 12 supervised weekly doses, and the inclusion of adult contacts in TITL, are feasible to implement at a programmatic level in Chile.

Key words: Latent tuberculosis; rifapentine; isoniazide; tuberculosis.

\section{Resumen}

Introducción: La prevención de la tuberculosis activa en los grupos de riesgo es clave para el control y eliminación de la tuberculosis. El tratamiento de la infección tuberculosa latente (TITL) con rifapentina e isoniazida en dosis semanales por 12 semanas es más corto que con otros esquemas, tiene menor hepatotoxicidad, mejor adherencia y es costo-efectivo. El objetivo del estudio es evaluar la factibilidad de implementar este esquema a nivel programático en Chile. Métodos: Se hizo una intervención piloto en territorios seleccionados entre mayo de 2018 y marzo de 2019. En esos territorios se reemplazó el esquema normado de TITL con isoniazida 6 meses por el esquema rifapentina-isoniazida 12 semanas. Además, se amplió la población objetivo, incluyendo a contactos mayores de 14 años. El tratamiento consistió en la administración conjunta de isoniazida y rifapentina por vía oral con frecuencia semanal, por 12 semanas, de forma supervisada por personal de salud. Resultados: Ingresaron 238 pacientes al piloto, de los cuales 53\% fueron mujeres y 54,2\% fueron mayores de 14 años. Del total de pacientes, 203 (85,3\%) completaron el tratamiento, 22 (9,2\%) lo abandonaron, 8 (3,4\%) presentaron reacciones adversas y 5 tuvieron otros motivos de egreso. Conclusión: Tanto el TITL con rifapentinaisoniazida por 3 meses en dosis semanales supervisadas, como la incorporación de contactos adultos a TITL, son factibles de implementar a nivel programático en Chile.

Palabras clave: Tuberculosis latente; rifapentina; isoniazida; tuberculosis.

* Programa de Control y Eliminación de la Tuberculosis, Ministerio de Salud, Gobierno de Chile. 


\section{Introducción}

La tuberculosis es en la actualidad la principal causa de muerte por un agente infeccioso en el mundo, produciendo más de 10 millones de enfermos y más de 1,5 millones de muertes cada año ${ }^{1}$. La infección por Mycobacterium tuberculosis es la etapa precursora de la enfermedad y se estima que un cuarto de la población mundial está infectada con el bacilo de la tuberculosis, es decir, aunque no presentan la enfermedad activa y no constituyen fuente de contagio, corresponden al reservorio de la enfermedad. A este estado de infección sin enfermedad se denomina tuberculosis latente ${ }^{2}$.

El $10 \%$ de las personas con tuberculosis latente desarrollará en algún momento de su vida la enfermedad activa. Se ha determinado que el riesgo de progresar a enfermedad es más alto en los primeros 2 años luego de la infección, pero se mantiene a lo largo de la vida ${ }^{2}$.

Desde el punto de vista de la salud pública, la prevención del desarrollo de tuberculosis activa en aquellos grupos poblacionales con un mayor riesgo de progresión es un elemento clave para el control de la tuberculosis, así como también para avanzar hacia la eliminación de esta enfermedad. La Organización Mundial de la Salud (OMS) establece que el diagnóstico y tratamiento de la tuberculosis latente, llamado también quimioprofilaxis, debe realizarse sistemáticamente en personas infectadas con el virus de la inmunodeficiencia humana (VIH), los contactos, niños $\mathrm{y}$ adultos, de casos de tuberculosis pulmonar, los enfermos de silicosis y los pacientes con comorbilidades o tratamientos que produzcan inmunosupresión ${ }^{3}$.

En Chile, la normativa vigente del Programa de Control y Eliminación de la Tuberculosis (PROCET) establece que el tratamiento de la infección tuberculosa latente (TITL) se debe realizar en los contactos menores de 15 años, los pacientes infectados con VIH y otros grupos de pacientes con inmunodeficiencias o infección tuberculosa reciente demostrada por el viraje de la prueba de tuberculina. El esquema de tratamiento definido corresponde a 6 meses de isoniazida diaria autoadministrada ( 9 meses para pacientes con $\mathrm{VIH})^{4}$. Por lo tanto, el TITL en contactos de 15 y más años es una intervención que no se realiza y que es necesario implementar.

Uno de los problemas del TITL con el esquema autoadministrado con isoniazida es la baja adherencia el tratamiento, lo que se produce especialmente en el caso de los adultos. Un gran porcentaje de los pacientes abandona la terapia antes de completar las dosis requeridas, debido a que el tratamiento es demasiado largo y a los efectos adversos que puede producir la isoniazida, entre ellos la hepatotoxicidad que es más frecuente en adultos $^{5,6}$. Se ha demostrado que esquemas más cortos que contiene rifamicinas tiene menos efectos adversos y mejoran la proporción de pacientes que completan el tratamiento ${ }^{7}$.

Entre los esquemas que usan rifamicinas, el esquema que combina rifapentina con isoniazida en una dosis diaria semanal por 12 dosis es uno de lo más recomendados por la OMS. La rifapentina es una rifamicina que tiene una vida media seis veces más prolongada que la rifampicina, lo que permite su administración una vez por semana. El uso de rifapentina asociado a isoniacida tiene varias ventajas con respecto a los otros esquemas: es un esquema de duración más corta (3 meses), ha demostrado producir menos hepatotoxicidad, tiene una eficacia similar al esquema de isoniazida por 9 meses y tiene una mejor adherencia al tratamiento. Además, la administración semanal permite realizarla en forma directamente supervisada por el personal de salud, asegurando una correcta administración y una evaluación más cercana del paciente ${ }^{3,8,9}$.

Desde el punto de vista de la relación costoefectividad, estudios llevados a cabo en Estados Unidos demostraron que el esquema con rifapentina era costo efectivo respecto a 9 meses de isoniazida, tanto desde la perspectiva del sistema de salud como de la sociedad ${ }^{10,11}$. Otros estudios realizados en escenarios de distinta carga de tuberculosis también han demostrado que el esquema con rifapentina es costo efectivo, aunque en países de bajos ingresos depende del precio de la rifapentina, el cual ha ido disminuyendo en los últimos años ${ }^{12-15}$.

En Chile, se realizó un estudio de costoefectividad comparando el uso del esquema con rifapentina directamente observado contra el escenario de mantener a los pacientes solo en seguimiento (en adultos) y contra el esquema de seis meses con isoniazida (en niños) concluyendo que el uso del esquema con rifapentina es costoefectivo en ambos casos ${ }^{16}$.

Considerando todos estos antecedentes, el PROCET realizó un estudio piloto para ofrecer tratamiento para tuberculosis latente con el esquema rifapentina más isoniazida, con el propósito de evaluar la adherencia al tratamiento al nuevo esquema, la aparición de reacciones adversas y la aplicación programática de esta intervención, con miras a realizar el cambio de la política de salud que actualmente se aplica en Chile. 


\section{Métodos}

Se llevó a cabo una intervención piloto en cuatro Servicios de Salud del país (de un total de 29), en el período comprendido entre mayo de 2018 y marzo de 2019. Los Servicios de Salud incluidos en el estudio se seleccionaron en base las características de su red asistencial, con el fin de incorporar redes con diferentes tipos de organización y disposición geográfica. Los Servicios de Salud (SS) seleccionados fueron: Metropolitano Oriente, Viña del Mar-Quillota, Concepción y Aysén.

En los territorios seleccionados se reemplazó el esquema con isoniazida normado para el TITL por el uso del esquema rifapentina-isoniazida $\mathrm{y}$, además, se incorporó a los contactos de 15 y más años al diagnóstico y tratamiento de la tuberculosis latente. Los fármacos requeridos para el esquema fueron provistos por el PROCET. Los exámenes $\mathrm{y}$ atenciones clínicas necesarias para cumplir el protocolo también fueron provistos por el programa en el contexto de sus prestaciones habituales.

La población a intervenir fueron personas con evidencia de infección por Mycobacterium tuberculosis, ya sea por PPD reactivo (prueba cutánea de tuberculina de $10 \mathrm{~mm}$ o más) o IGRA (ensayo de liberación de interferón gama) positivo, y en quienes se hubiese descartado la enfermedad tuberculosa activa. Los criterios de inclusión y exclusión son los descritos en la Tabla 1.

El tratamiento consistió en la administración conjunta de isoniazida y rifapentina por vía oral en las dosis descritas en la Tabla 2, con frecuencia semanal, por 12 semanas (total 12 dosis). La administración de los fármacos se realizó de forma supervisada por personal de salud capacitado, debiendo el paciente acudir semanalmente a un centro de su salud para la administración.

Para las personas que no pudieron utilizar este esquema de tratamiento debido a criterios de exclusión, pero que sí tenían indicación de quimioprofilaxis según la normativa vigente, el TITL se mantuvo con el esquema de isoniazida por 6 meses autoadministrada.

Todos los pacientes incluidos en el protocolo tuvieron controles médicos mensuales. Las reacciones adversas a fármacos fueron manejadas de acuerdo a lo establecido por la Norma Técnica del PROCET para sospecha de reacción adversa medicamentosa en tratamiento de tuberculosis o quimioprofilaxis ${ }^{4}$.

En el caso de que un paciente no acudiera a la toma de tratamiento en el día programado, se realizaron llamadas telefónicas dentro del mismo día de la inasistencia y visitas al domicilio dentro de la misma semana, para prevenir el abandono del tratamiento.

Toda persona incluida en el protocolo recibió una educación individual al ingreso, dirigida a informarle sobre el tratamiento, la importancia de su adherencia a las dosis programadas, y sobre los síntomas y signos de eventuales efectos

Tabla 1. Criterios de inclusión y exclusión para el tratamiento de la infección tuberculosa latente con nuevo esquema

\section{Inclusión}

Contactos intradomiciliarios de pacientes con tuberculosis pulmonar con bacteriología positiva, que tengan entre 2 y 50 años de edad, con evidencia de infección tuberculosa y descartada la tuberculosis activa

Pacientes inmunodeprimidos con evidencia de infección tuberculosa en quienes se haya descartado tuberculosis activa

Otros sujetos mayores de dos años que tengan indicación de quimioprofilaxis para tuberculosis y en quienes se haya descartado tuberculosis activa

\section{Exclusión}

Personas con intolerancia o hipersensibilidad a la rifapentina o la isoniazida

Personas que hayan recibido tratamiento antituberculoso anteriormente

Menores de 2 años o mayores de 50 años de edad

Contactos con PPD $<10 \mathrm{~mm}$

Embarazadas

Personas en tratamiento antirretroviral por VIH

Personas con antecedentes de enfermedad hepática crónica o alcoholismo activo

Personas que no puedan cumplir con la asistencia a un centro de salud

Contactos de pacientes con tuberculosis resistente a isoniazida y/o rifampicina

Tabla 2. Dosis semanales de rifapentina e isoniazida para el esquema de tratamiento de la infección tuberculosa latente

\begin{tabular}{|cccc|}
\hline \multicolumn{2}{|c}{ Rifapentina* } & \multicolumn{2}{c|}{ Isoniazida } \\
$\begin{array}{c}\text { Peso corporal } \\
\text { (kg) }\end{array}$ & $\begin{array}{c}\text { Dosis } \\
(\mathbf{m g})\end{array}$ & $\begin{array}{c}\text { Menores de } \\
\mathbf{1 2} \text { años }\end{array}$ & $\begin{array}{c}\mathbf{1 2} \text { años y } \\
\text { más }\end{array}$ \\
$10-14$ & 300 & & \\
$14,1-25$ & 450 & & \\
$25,1-32$ & 600 & $25 \mathrm{mg} / \mathrm{kg}$ & $15 \mathrm{mg} / \mathrm{kg}$ \\
$32,1-50$ & 750 & & \\
$>50$ & 900 & & \\
\hline
\end{tabular}

*Tabletas de $150 \mathrm{mg}$. 
adversos. Debido a las características propias del Programa de Tuberculosis y a su normativa, el TITL en menores de 18 años es obligatorio, pero no así en los sujetos adultos, quienes pueden decidir tratarse o no tratarse. Estos mismos criterios se usaron para clasificar los resultados del nuevo esquema de tratamiento (Tabla 3 ).

Se analizaron indicadores estadísticos descriptivos para la mayoría de los datos obtenidos, y, cuando fue necesario, se aplicó el test Chi cuadrado para comparar los grupos con un nivel de significancia de 0,05 .

\section{Resultados}

En el período comprendido entre mayo de 2018 y marzo de 2019 ingresaron en total 238 pacientes al estudio piloto, distribuidos geográficamente de la siguiente manera: SS Metropolitano Oriente: 94, SS Viña del Mar-Quillota: 109, SS Concepción: 32 y SS Aysén: 3.

El $53 \%$ de las personas ingresadas a trata- miento fueron mujeres. 54,2\% fueron personas de 15 y más años (adultos) cuya edad promedio fue de 38 años. La distribución de los pacientes ingresados según la causa del tratamiento fue la siguiente: $109(45,8 \%)$ personas fueron contactos intradomiciliarios menores de 15 años de edad, $82(34,5 \%)$ fueron contactos intradomiciliarios de 15 o más años, $30(12,6 \%)$ fueron adultos no contactos con condiciones inmunosupresoras, y $17(7,1 \%)$ fueron adultos privados de libertad (criterio de inclusión $\mathrm{N}^{\circ} 3$ : indicación de quimioprofilaxis por otra causa). De total de pacientes que entraron al protocolo, $203(85,3 \%)$ cumplieron criterio de alta con tratamiento completo. Se registraron $22(9,2 \%)$ abandonos de tratamiento, $8(3,4 \%)$ reacciones adversas que llevaron a suspensión del tratamiento, 1 caso de traslado de región sin información de egreso, y 4 casos en los cuales se suspendió el tratamiento por indicación médica de causa distinta a reacción adversa medicamentosa. No se registraron fallecidos en ninguno de los grupos (Figura 1).

\section{Tabla 3. Definiciones para el resultado de tratamiento}

\begin{tabular}{|ll|}
\hline Resultado & Definición \\
\hline Alta & $\begin{array}{l}\text { Paciente que completa las } 12 \text { dosis semanales en un plazo máximo de } 16 \\
\text { semanas }\end{array}$ \\
\hline $\begin{array}{l}\text { Traslado sin información de egreso } \\
\text { Fallecido }\end{array}$ & $\begin{array}{l}\text { Paciente que deja de acudir a tratamiento por } 4 \text { semanas seguidas } \\
\text { rante el tratamiento, pero no es posible trazar el término de su tratamiento }\end{array}$ \\
\hline $\begin{array}{l}\text { Reacción adversa a medicamentos } \\
\text { (RAM) }\end{array}$ & $\begin{array}{l}\text { Paciente que fallece durante el tratamiento sin llegar a completarlo, indepen- } \\
\text { diente de la causa de muerte }\end{array}$ \\
$\begin{array}{l}\text { Suspensión del tratamiento por otra } \\
\text { completarlo } \\
\text { causa }\end{array}$ & $\begin{array}{l}\text { Situación en la cual el médico tratante decide suspender el tratamiento antes } \\
\text { de completarlo, por una causa clínica que no es una RAM }\end{array}$ \\
\hline
\end{tabular}

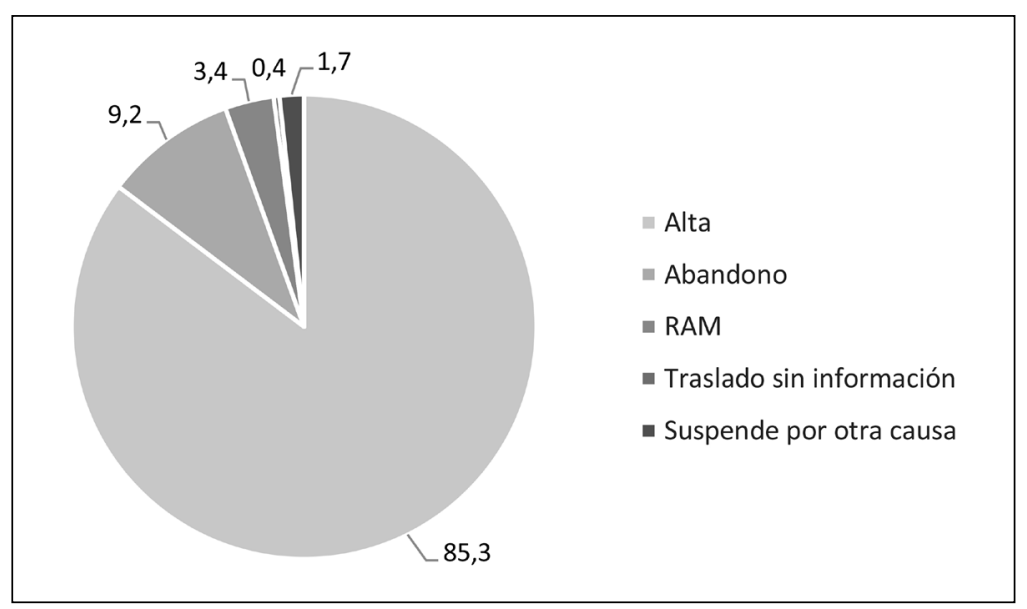

Figura 1. Resultados de tratamiento $(\%)$ del total de pacientes $(n=238)$. $85,3 \%$ de los pacientes cumplieron el tratamiento completo. 
Los resultados de tratamiento según edad muestran que el grupo de 15 años o más tiene un mayor porcentaje de altas $(87,6 \%)$ respecto al grupo pediátrico $(82,6 \%)$, aunque esta diferencia no es estadísticamente significativa $\left(\mathrm{p}=0,27 ; \chi^{2}\right)$. Así mismo, los hombres tienen mejores resultados que las mujeres en ambos grupos de edad, aunque tampoco llega a ser estadísticamente significativo $\left(\mathrm{p}=0,36 ; \chi^{2}\right)$. De todas maneras, llama la atención el mayor porcentaje de abandono que se produce en mujeres respecto de los hombres (Tabla 4).

La suspensión de tratamiento debido a reacciones adversas es baja, pero más frecuente en mujeres, las que alcanzan un 4,8\%. La suspensión del tratamiento por otras causas se dio solamente en el grupo de hombres menores de 15 años, correspondiendo a 4 casos, dos de los cuales se suspendieron porque se descartó la tuberculosis en el caso índice y otros dos debido a razones no especificadas por los tratantes.

En la Tabla 5 se muestran los resultados del tratamiento según el Servicio de Salud. El porcentaje de altas es similar para los tres SS que ingresaron mayor cantidad de pacientes. El SS Aysén solo ingresó 3 pacientes a tratamiento, uno de los cuales lo debió suspender por RAM (reacción adversa a medicamentos). El porcentaje de abandono de tratamiento es superior en el SS Viña del Mar-Quillota, y la totalidad del ítem 'suspensión de tratamiento por otra causa' se registró en el SS Metropolitano Oriente.

En la Tabla 6 se muestran los resultados del TITL según la causa de ingreso a tratamiento.

Tabla 4. Resultados del tratamiento de la infección tuberculosa latente obtenidos en grupos por edad y sexo

\begin{tabular}{|lrrrcccc|}
\hline & Total & Altas & Abandonos & RAM & $\begin{array}{c}\text { Suspensión por } \\
\text { otra causa }\end{array}$ & $\begin{array}{c}\text { Traslado } \\
\text { sin info.* }\end{array}$ & Fallecidos \\
Menores de 15 años & 109 & $90 ; 82,6 \%$ & $10 ; 9,2 \%$ & $4 ; 3,7 \%$ & $4 ; 3,7 \%$ & $1 ; 0,9 \%$ & 0 \\
Hombres & 54 & $45 ; 83,3 \%$ & $3 ; 5,6 \%$ & $1 ; 1,9 \%$ & $4 ; 7,4 \%$ & $1 ; 1,9 \%$ & 0 \\
Mujeres & 55 & $45 ; 81,8 \%$ & $7 ; 12,7 \%$ & $3 ; 5,5 \%$ & 0 & 0 & 0 \\
15 años o más & 129 & $113 ; 87,6 \%$ & $12 ; 9,3 \%$ & $4 ; 3,1 \%$ & 0 & 0 & 0 \\
Hombres & 58 & $53 ; 91,4 \%$ & $4 ; 6,9 \%$ & $1 ; 1,7 \%$ & 0 & 0 & 0 \\
Mujeres & 71 & $60 ; 84,5 \%$ & $8 ; 11,3 \%$ & $3,4,2 \%$ & 0 & 0 & 0 \\
Total hombres & 112 & $98 ; 87,5 \%$ & $7 ; 6,3 \%$ & $2 ; 1,8 \%$ & $4 ; 3,6 \%$ & $1 ; 0,9 \%$ & 0 \\
Total mujeres & 126 & $105 ; 83,3 \%$ & $15 ; 11,9 \%$ & $6 ; 4,8 \%$ & 0 & 0 & 0 \\
Total & 238 & $20385,3 \%$ & $22 ; 9,2 \%$ & $8 ; 3,4 \%$ & $4 ; 1,7 \%$ & $1 ; 0,4 \%$ & 0 \\
\hline
\end{tabular}

*Traslado sin info.: Traslado sin información.

Tabla 5. Resultados del tratamiento de la infección tuberculosa latente obtenidos según Servicio de Salud

\begin{tabular}{|lccccccc|}
\hline Servicio de salud & Total & Alta & Abandono & RAM & $\begin{array}{c}\text { Traslados } \\
\text { sin } \\
\text { información }\end{array}$ & $\begin{array}{c}\text { Suspensión } \\
\text { por otra } \\
\text { causa }\end{array}$ & Fallecido \\
Viña del Mar - Quillota & 109 & $94 ; 86,2 \%$ & $14 ; 12,8 \%$ & $1 ; 0,9 \%$ & 0 & 0 & 0 \\
Metropolitano Oriente & 94 & $80 ; 85,1 \%$ & $5 ; 5,3 \%$ & $4 ; 4,3 \%$ & $1 ; 1,1 \%$ & $4 ; 4,3 \%$ & 0 \\
Concepción & 32 & $27 ; 84,4 \%$ & $3 ; 9,4 \%$ & $2 ; 6,3 \%$ & 0 & 0 & 0 \\
Aysén & 3 & $2 ; 66,7 \%$ & 0 & $1 ; 33,3 \%$ & 0 & 0 & 0 \\
\hline
\end{tabular}

Tabla 6. Resultados del tratamiento de la infección tuberculosa latente obtenidos según causa de indicación del tratamiento

\begin{tabular}{|lrrrrccc|}
\hline & Total & Altas & Abandonos & RAM & $\begin{array}{c}\text { Suspensión } \\
\text { por otra } \\
\text { causa }\end{array}$ & $\begin{array}{c}\text { Traslado } \\
\text { sin } \\
\text { información }\end{array}$ & $\begin{array}{c}\text { Fallecidos } \\
\text { Contactos menores de 15 años }\end{array}$ \\
& 109 & $90 ; 82,6 \%$ & $10 ; 9,2 \%$ & $4 ; 3,7 \%$ & $4 ; 3,7 \%$ & $1 ; 0,9 \%$ & 0 \\
Contactos de 15 años o más & 82 & $71 ; 86,6 \%$ & $8 ; 9,8 \%$ & $3 ; 3,7 \%$ & 0 & 0 & 0 \\
Inmunosuprimidos & 30 & $27 ; 90 \%$ & $2 ; 6,7 \%$ & $1 ; 3,3 \%$ & 0 & 0 & 0 \\
Otra causa (privados de libertad) & 17 & $15 ; 88,2 \%$ & $2 ; 11,8 \%$ & 0 & 0 & 0 & 0 \\
\hline
\end{tabular}


Las cohortes de tratamiento separadas por causa de indicación de TITL muestran el porcentaje más alto de éxito en los pacientes adultos inmunosuprimidos, quienes alcanzaron un $90 \%$ de altas y solo un $6,7 \%$ de abandonos. Sin embargo, ninguna de las diferencias fue estadísticamente significativa.

Si se comparan los grupos por edad entre los contactos, los menores de 15 años tienen un porcentaje de altas inferior $(82,6 \%)$ respecto a los adultos $(86,6 \%)$, aunque esta diferencia tampoco es estadísticamente significativa $\left(p=0,45 ; \chi^{2}\right)$. Cabe destacar que esta diferencia se dio a expensas principalmente de la suspensión del tratamiento por otra causa.

Los privados de libertad alcanzaron un alto porcentaje de altas $(88,2 \%)$ pero también los mayores porcentajes de abandono $(11,8 \%)$. Estos abandonos se producen cuando los pacientes salen en libertad.

En relación a las reacciones adversas a medicamentos que llevaron a suspensión del esquema, se reportaron 5 casos de intolerancia digestiva que no respondieron a manejo sintomático (4 niños y un adulto), un caso adulto de hepatotoxicidad, un caso adulto de reacción alérgica cutánea moderada, y un caso adulto de síndrome $f u$-like (pseudogripal).

\section{Discusión}

Las ventajas del esquema de tratamiento para la infección tuberculosa latente con rifapentina/ isoniazida han sido ampliamente documentadas por distintas investigaciones a nivel mundial y el esquema ya es recomendado como uno de los preferentes por diversos organismos internacionales, entre ellos la propia $\mathrm{OMS}^{3}$. De hecho, este esquema ha sido fuertemente recomendado para adultos y niños sobre los dos años, incluyendo los pacientes con VIH cuando las interacciones farmacológicas los permitan ${ }^{17}$. En este estudio piloto los pacientes con VIH fueron excluidos para evitar interferencias en la evaluación de la efectividad programática de la intervención; sin embargo, este grupo debe ser incluido para el uso de este esquema cuando los fármacos antirretrovirales que cada individuo utiliza lo permita.

Este ensayo piloto desarrollado por el PROCET en Chile permitió observar como esta intervención puede ser implementada a nivel programático, considerando tanto el cambio del esquema en pacientes que tienen actualmente la indicación de TITL según la normativa, como también la incorporación de un nuevo grupo de pacientes que deben ser sometidos a esta inter- vención, como son los contactos adultos de los casos de tuberculosis pulmonar con bacteriología positiva.

El estudio mostró que existe una alta adherencia al tratamiento con el nuevo esquema, siendo las altas de tratamiento de un $85,3 \%$ en forma global con solo un $9,2 \%$ de abandonos. Esta proporción de personas que adhieren al tratamiento y que lo completan es similar o más alta que la reportada en estudios en otros países. Por ejemplo, un estudio multicéntrico realizado el año 2017 mostró que un $87,2 \%$ de los pacientes $(83,1 \%$ a $90,5 \%$; IC 95\%) completaron el tratamiento en el grupo en que se realizó en forma directamente supervisada $^{18}$. Otro estudio realizado en Nuevo México y publicado en febrero de 2020, reportó una adherencia de $75 \%$ en un grupo de 40 pacientes a quienes se les realizó TITL directamente observado con el mismo régimen ${ }^{19}$. El estudio de Sterling et al. ${ }^{8}$ del año 2011 reportó una adherencia de $82,1 \%$.

Sin embargo, la comparación de la adherencia al tratamiento con lo que se reporta por otros estudios internacionales es compleja, debido a la heterogeneidad de las definiciones y de las condiciones bajo las cuales se realiza la intervención, por ejemplo, si el tratamiento es directamente observado o autoadministrado. Un metaanálisis publicado el año 2017 da cuenta de esta heterogeneidad, aunque aun así estima que esta adherencia es mayor que con los regímenes con isoniazida por 6 o 9 meses $^{20}$.

En el caso de Chile, esta comparación entre el nuevo esquema y la adherencia al esquema autoadministrado con isoniazida no es posible de realizar debido, primero, a que el tratamiento de contactos de 15 y más años es una intervención nueva, y en segundo lugar porque la adherencia al tratamiento con isoniazida autoadministrada en contactos menores de 15 años no ha sido monitorizada por el nivel central del programa de tuberculosis.

Llama la atención en los resultados los 10 casos de abandono de tratamiento en los contactos menores de 15 años, considerando que los equipos de tuberculosis tienen la indicación y la facultad legal de hacer cumplir esta terapia en este grupo ${ }^{4}$. A nivel internacional, la adherencia a tratamiento en niños con el régimen rifapentina/ isoniazida puede llegar hasta más del $95 \%$, especialmente si se toman estrategias de tipo sociales para evitar el abandono ${ }^{21,22}$. En el diseño de este ensayo piloto no estaba contemplado realizar la auditoría de abandono que se realiza en el caso de pérdida de seguimiento en el tratamiento de la tuberculosis activa, por lo que no es posible 
conocer a cabalidad el perfil de las personas que abandonaron el tratamiento. Esta estrategia sería importante de incluir cuando la intervención se realice en forma normada por el programa, especialmente en los casos infantiles.

Con respecto a las reacciones adversas, el estudio piloto realizado muestra un porcentaje de $3,4 \%$ de reacciones adversas que obligaron a suspender tratamiento, las que se presentaron con igual frecuencia en adultos y en niños. Las reacciones adversas fueron principalmente la intolerancia digestiva, y solo se produjo un caso de hepatotoxicidad, lo que corresponde a un $0,4 \%$ del total de personas intervenidas. La hepatotoxicidad reportada con este esquema de tratamiento a nivel internacional es de $0,36 \%(0,18-0,73)^{8,17}$.

En el estudio de Sterling et al. ${ }^{8}$ del año 2011 se reportó una suspensión del tratamiento debido a RAM de un 4,9\%. Un análisis posterior realizado con los datos obtenidos de este mismo estudio da cuenta de que un 3,5\% de los pacientes tuvieron una RAM sistémica con el esquema rifapentina/ isoniazida semanal, especialmente síndrome flulike, aunque en la mayoría de ellos los efectos adversos no se consideraron graves, sí obligaron a, al menos, cambiar el tratamiento ${ }^{23}$.

Es probable que el riesgo de desarrollar una RAM a este régimen combinado esté relacionado con factores genéticos. Estudios realizados en población oriental muestran altos porcentajes de RAM, especialmente síndrome $f l u$-like en países como Taiwan y Corea ${ }^{24,25}$. En el caso de los latinos, el estudio de Sterling et al. ${ }^{23}$ del año 2015 mostró que los latinos tenían menos efectos adversos sistémicos que los blancos no hispanos, lo que podría explicar los bajos porcentajes de RAM de tipo sistémicos producidos en este ensayo.

Así mismo, las RAM al esquema semanal con rifapentina/isoniazida son más frecuentes en personas de mayor edad y en mujeres. Una de las limitaciones de este estudio está dada por la edad máxima para acceder al esquema, que fue de 50 años, criterio que no se utilizó en los estudios internacionales revisados, por lo tanto, los efectos adversos podrían estar subestimados. En el caso de las diferencias por sexo, las mujeres tuvieron 2,6 veces más reacciones adversas que los hombres, lo que se condice con lo reportado en los estudios internacionales ${ }^{8,23}$. La presencia de mayores reacciones adversas en mujeres podría explicar el mayor porcentaje de abandonos que se produjo en este grupo, situación que llama la atención considerando que normalmente los abandonos de tratamiento se producen en los hombres ${ }^{26}$.

Una limitación importante al desarrollo del en- sayo piloto fue que el reclutamiento de las personas fue menor al esperado, debido principalmente al rechazo por parte de los clínicos a ofrecer este nuevo esquema. Por una parte, los pediatras se resistieron al cambio de terapia para los menores de 15 años y, por otra parte, la necesidad de que la indicación de TITL para los adultos se hiciera desde los niveles secundarios constituye una barrera de acceso para estos pacientes. Aunque es comprensible que se produzcan estas resistencias, probablemente se requiere de una mejor comunicación de la evidencia y de una gestión del cambio más eficiente para instalar esta terapia a nivel programático.

\section{Conclusión}

El estudio desarrollado muestra que el tratamiento para tuberculosis latente con el esquema rifapentina más isoniazida en dosis semanal es factible de realizar a nivel programático, tiene una buena adherencia al tratamiento, tanto en adultos como en niños, y los efectos adversos reportados son escasos. Por lo tanto, se recomienda realizar el cambio de la política de salud que actualmente se aplica en Chile.

Nota: A la fecha, el Programa de Control y Eliminación de la Tuberculosis ha incluido el esquema evaluado en este estudio piloto como el tratamiento prioritario para la tuberculosis latente, ampliando los criterios de inclusión y asegurando el abastecimiento y la cobertura a nivel nacional.

\section{Bibliografía}

1.- WORLD HEALTH ORGANIZATION. Global tuberculosis report 2018. Geneva. World Health Organization, 2019.

2.- HOUBEN R, DODD P. The Global Burden of Latent Tuberculosis Infection: a re-estimation using mathematical modelling. PLOS Med. 2016. 13 (10): e1002152. https://doi.org/10.1371/journal.pmed.1002152.

3.- ORGANIZACIÓN MUNDIAL DE LA SALUD. Directrices sobre la atención de la infección tuberculosa latente. 2015.

4.- PROGRAMA NACIONAL DE CONTROL Y ELIMINACIÓN DE LA TUBERCULOSIS. Normas técnicas para el control y la eliminación de la tuberculosis [Internet]. 2014. [Consultado el 7 de febrero de 2020]. Disponible en: http://web.minsal.cl/sites/default/files/ NORMA_TECNICA_TUBERCULOSIS.pdf

5.- VÉJAR L, HENAO M. Adherencia a quimioprofilaxis de niños chilenos expuestos a tuberculosis del adulto. Rev Chil Enferm Respir. 2006; 22: 164-7. 
6.- PALMERO D. ¿Un nuevo esquema de quimioprofilaxis en tuberculosis? Rev Am Med Resp. 2012; 1: 17-19.

7.- VILLA S, FERRAREZE M, SOTGIE G, CASTELLOTTI P, SADERI L, GRECCHI C, et al. Latent tuberculosis infection treatment completion while shifting prescription from isoniazid only to rifampicin.contained regimens: a two decade experience in Milan, Italy. J Clin Med. tuberculosis [Internet]. 2020. [Consultado el 7 de febrero de 2020]. Disponible en: https://www.mdpi. com/2077-0383/9/1/101/htm

8.- STERLING T, VILLARINO E, BORISOV A, SHANG N, GORDIN F, BLIVEN-SIZEMORE, et al. Three months of rifapentine and isoniazid for latent tuberculosis infection. New Engl J. Med 2011; 365 (23): 2155-66.

9.- VILLARINO ME, SCOTT NA, WEIS SE, WEINER M, CONDE MB, JONES B, et al. Treatment for preventing tuberculosis in Children and adolescents: A randomized clinical trial of a 3-Month, 12-Dose Regimen of a combination of rifapentine and isoniazid. JAMA Pediatr [Internet]. 2015 [consultado el 7 de febrero de 2020]. Disponible en: http://archpedi.jamanetwork.com/article. aspx? articleid $=2089639$

10.- SHEPARDSON D, MARKS SM, CHESSON H, KERRIGAN A, HOLLAND DP, SCOTT N, et al. Costeffectiveness of a 12-dose regimen for treating latent tuberculous infection in the United States. Int J Tuberc Lung Dis. 2013; 17: 1531-7.

11.- SHEPARDSON D, KENZIE WI. Update on costeffectiveness of a 12-dose regimen for latent tuberculous infection at new rifapentine prices [Internet]. Int J Tuberc Lung Dis. 2014; 18 (6): 751-3.

12.- HUANG YW, YANG SF, YEH YP, TSAO TC, TSAO SM. Impacts of 12-dose regimen for latent tuberculosis infection: treatment completion rate and cost effectiveness in Taiwan. Medicine. 2016; 95 (34): 1-5.

13.- DENHOLM JT, MCBRYDE ES, EISEN D, STREET A, MATCHETT E, CHEN C, et al. SIRCLE: a randomised controlled cost comparison of self-administered short-course isoniazid and rifapentine for cost effective latent tuberculosis eradication. Intern Med J. 2017; 47: 1433-6.

14.- JOHNSON KT, CHURCHYARD GJ, SOHN H, DOWDY DW. Cost- effectiveness of preventive therapy for tuberculosis with isoniazid and rifapentine versus isoniazid alone in high-burden settings. Clin Infect Dis. 2018; 67: 1072-8.

15.- DOAN T, FOX G, MEEHAN M, SCOTT N, RAGONNET R, VINEY K, et al. Cost-effectiveness of 3 months of weekly rifapentine and isoniazid compared with other standard treatment regimens for latent tuberculosis infection: a decision analysis study. J. Antimicrob Chemother 2019; 7: 218-27.

16.- HERRERA T. Costo efectividad del tratamiento de la tuberculosis latente con rifapentina/isoniacida a contactos en Chile. [Tesis de magíster]. Escuela de Salud Pública, Universidad de Chile. 2017. 76 p.
17.- STERLING T, NJIE G, ZENNER D, COHN D, REVES R, AHMED A, et al. Guidelines for the treatment of latent tuberculosis infection: recommendations from the National Tuberculosis Controllers Association and CDC, 2020. MMWR Recomm Rep. 2020; 69 (1): 1-11.

18.- BELKNAP R, HOLLAND D, FENG PJ, MILLET JP, CAYLÀ J, MARTINSON N, et al. Self-administered versus Directly Observed Once-Weekly Isoniazid and Rifapentine Treatment of Latent Tuberculosis Infection: A Randomized Trial. Ann Intern Med. 2017; 167: 68997.

19.- JACKEMAN B, LOGOTHETIS S, ROBERTS M, BACHYRYCS A, FORTUNE D, BURGOS M. Addressing latent tuberculosis infection treatment through a collaborative care model with community pharmacies and a health department. Prev Chron Dis. 2020; 17: 1-9.

20.- PEASE C, HUTTON B, YAZDI F, WOLFE D, HAMEL C, QUACH P, et al. Efficacy and completion rates of rifapentine and isoniazid (3HP) compared to other treatment regimens for latent tuberculosis infection: a systematic review with netwotk meta-analyses. BMC Infect Dis. 2017; 17 (265): 1-11.

21.- SANTOS JC, SILVA JB, RANGEL MA, BARBOSA L, CARVALHO I. Preventive therapy compliance in pediatric tuberculosis - a single center experience. Pulmonology. 2020; 26: 78-83.

22.- CRUZ A, STARKE J. Safety and adhrence for 12 weekly doses of Isoniazid and Rifapentine for pediatric tuberculosis infection. Ped Infect Dis J. 2016; 35: 81113.

23.- STERLING T, MORO R, BORISOV A, PHILLIPS E, SHEPHERD G, ADKINSON N, et al. Flu-like and other systemic drug reactions among persons receiving weekly rifapentine plus isoniazid or daily isoniazid for treatment of latent tuberculosis infection in the PREVENT tuberculosis study. Clin Infect Dis. 2015; 61: 527-35.

24.- JEON D. Latent tuberculosis infection: recent progress and challenges in South Korea. Korean J Intern Med. 2020; 35: 269-75.

25.- YU Y-Y, TSAO S-M, YANG W-T, HUANG W-C, LIN C-H. CHEN W-W, et al. Association of Drug Metabolic Enzyme Genetic Polymorphisms and Adverse Drug Reactions in Patients Receiving Rifapentine and Isoniazid Therapy for Latent Tuberculosis. Int. J. Environ. Res. Public Health. 2020; 17: 210-8.

26.- TORRES Z, HERRERA T. Perfil del paciente con tuberculosis que abandona el tratamiento en Chile. Rev Chil Enferm Respir. 2015; 31: 52-7.

Correspondencia a:

Dra. Nadia Escobar Salinas

Programa de Control y Eliminación de la Tuberculosis, Ministerio de Salud. Gobierno de Chile.

Email: nadia.escobar.s@gmail.com 